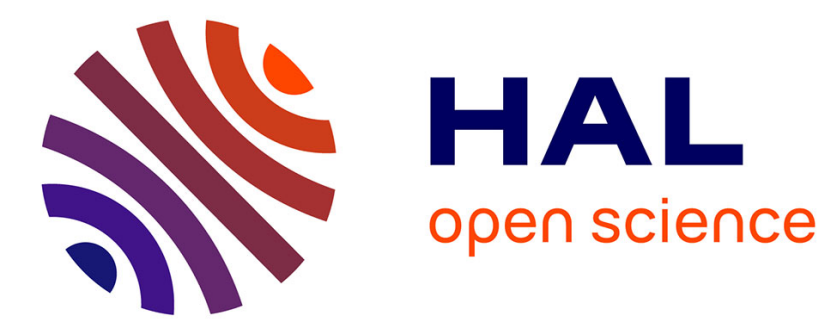

\title{
Efficient access to enantiomerically pure rigid diamines
}

\author{
Loïc Planas, Joelle Pérard-Viret, Jacques Royer
}

\section{To cite this version:}

Loïc Planas, Joelle Pérard-Viret, Jacques Royer. Efficient access to enantiomerically pure rigid diamines. Tetrahedron: Asymmetry, 2004, 15 (15), pp.2399-2403. 10.1016/j.tetasy.2004.06.011 . hal03385819

\section{HAL Id: hal-03385819 \\ https://hal.science/hal-03385819}

Submitted on 19 Oct 2021

HAL is a multi-disciplinary open access archive for the deposit and dissemination of scientific research documents, whether they are published or not. The documents may come from teaching and research institutions in France or abroad, or from public or private research centers.
L'archive ouverte pluridisciplinaire HAL, est destinée au dépôt et à la diffusion de documents scientifiques de niveau recherche, publiés ou non, émanant des établissements d'enseignement et de recherche français ou étrangers, des laboratoires publics ou privés. 


\title{
Efficient access to enantiomerically pure rigid diamines
}

\author{
Loïc Planas, Joëlle Pérard-Viret and Jacques Royer* \\ Synthèse et structure de molécules d'intérêt pharmacologique (UMR 8638 CNRS-Université René Descartes), \\ Faculté des Sciences Pharmaceutiques et Biologiques, 4 avenue de l'Observatoire, 75270 Paris Cedex 06, France
}

\begin{abstract}
Asymmetric spiro cyclization of a pyrrolidine derivative was used as a key step for constructing novel rigid diamines. A selected example has proved its utilitv as a chiral nonmetallic catalyst in a Michael reaction.
\end{abstract}

\section{Introduction}

Chiral diamines ${ }^{1}$ are largely used in asymmetric processes as chiral bases, ${ }^{2}$ chiral ligands, ${ }^{3}$ and chiral catalysts. ${ }^{4}$ In recent years, increasing interest has been given to enantioselective reactions catalyzed by nonmetallic compounds. ${ }^{5}$ Several natural products, such as the well known alkaloid sparteine, have been long recognized to be efficient reagents in asymmetric deprotonation processes and asymmetric reactions in general. ${ }^{6}$ The main problem usually arising with natural products is the occurrence of only one enantiomer $;^{7}$ new synthetic asymmetric diamines, available in both enantiomeric forms, are thus still interesting to design, prepare, and investigate. For example, bipyrrolidines $\mathbf{1}^{8}$ and $\mathbf{2}^{9}$ (Fig. 1) can be used for the enantioselective Michael addition of carbonyl compounds to nitroalkenes. Starting from these interesting results, we envisioned preparing similar compounds containing a highly constrained structure as 3, easily accessible from the already described spiro compound 4.

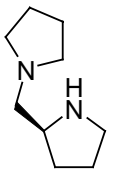

1

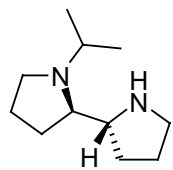

2

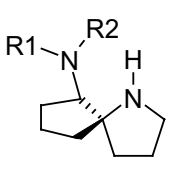

3

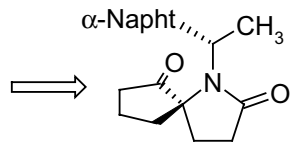

4
Figure 1.

\footnotetext{
* Corresponding author. Tel.: +33-1-537-397-49; fax: +33-1-432-91403; e-mail: jacques.royer@pharmacie.univ-paris5.fr
}

\section{Results and discussion}

We recently reported an original asymmetric access to the 1-azaspiro[4.4]nonane skeleton ${ }^{10}$ using the chemistry of chiral nonracemic $\alpha, \beta$-unsaturated $\gamma$-lactams. ${ }^{11}$ Compound 4 was obtained in 35\% overall yield from $(S)$-naphthylethylamine through the four-step sequence shown in Scheme 1. ${ }^{10}$

With 1-azaspiro[4.4]nonane 4 in hand, we planned to synthesize chiral diamines $\mathbf{3}$ by the use of simple reactions allowing rapid access to the target compounds. In order to develop an efficient preparation of several diamines, we first focused on the primary amine $\mathbf{1 0}$ (see Scheme 2), which could be further transformed to variously substituted compounds. 1-Azaspiro[4.4]nonane2,6-dione 4, was treated with benzylamine in refluxing toluene and in the presence of $\mathrm{TsOH}$ and the crude reaction mixture, which consisted of a 1:1 mixture of imine $8 \mathbf{a}$ and enamine $\mathbf{8 b}$, was reduced by $\mathrm{NaBH}_{4}$ in methanol to furnish, in a quantitative yield over the two steps, amine 9 as a unique stereoisomer (Scheme 2). The $N$-benzyl group was easily cleaved by simple hydrogenolysis to furnish primary amine $\mathbf{1 0}$ in $81 \%$ yield.

From this building block, numerous diamines can be prepared. Focusing on the access to a new rigid bipyrrolidine, we converted this primary amine to the corresponding succinimide $\mathbf{1 1}$ through treatment with succinic anhydride and acetyl chloride. The exhaustive reduction of 11 using borane in THF furnished $\mathrm{N}$-protected diamine 12, which was hydrogenolyzed to give target diamine $\mathbf{1 3}$ in $76 \%$ yield after purification.

Following a method developed by Riguera et al., ${ }^{12}$ the absolute configuration of the newly formed stereogenic 

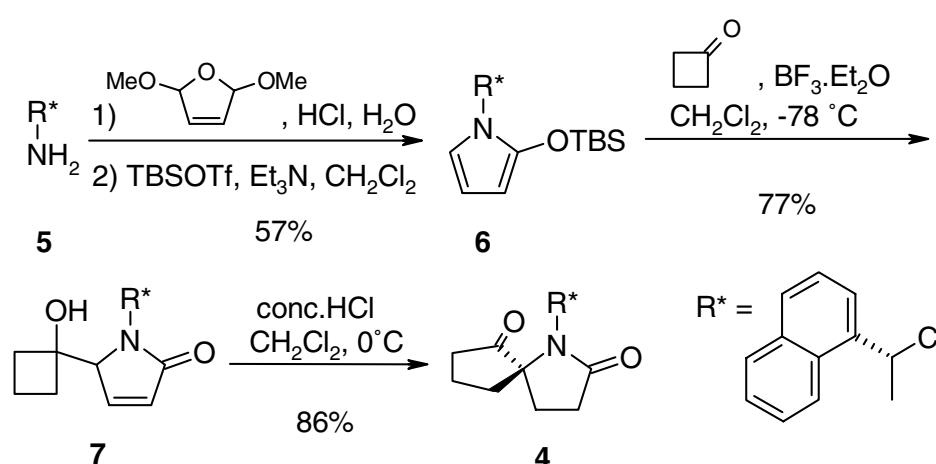<smiles>CC(C)c1cccc2ccccc12</smiles>

Scheme 1.

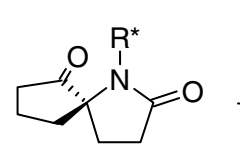

4

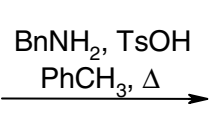<smiles>[R7]N1C(=O)CC[C@]12CCC/C2=N/Br</smiles>

8a

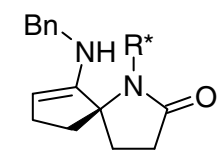

$8 b$

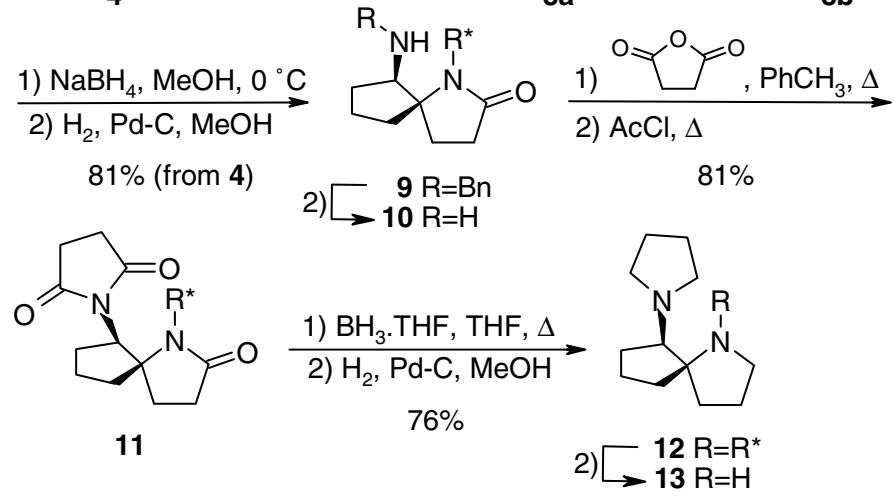

Scheme 2.

center in 10, was deduced from the ${ }^{1} \mathrm{H}$ NMR investigation of both diastereoisomers 14a and 14b formed through the coupling of primary amine 10, respectively, with $(R)$ - and $(S)$ - $N$-Boc-phenylglycine (Scheme 3 ).

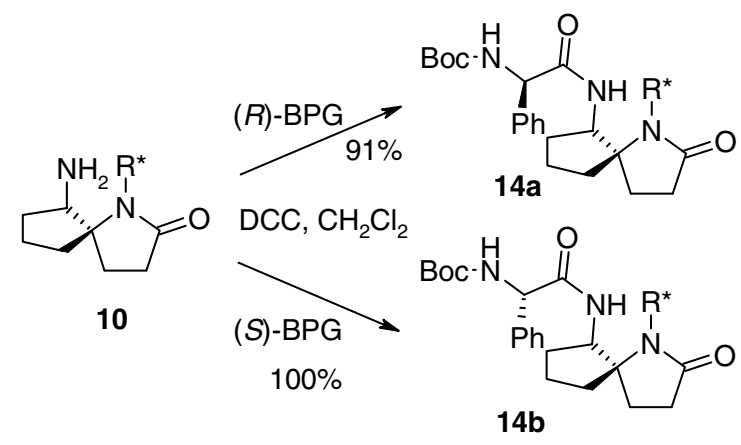

Scheme 3.

Observation of the variation in the chemical shifts of both diastereoisomers 14a and 14b showed either positive $\Delta \delta^{(R, S)}$ on one side, or negative on the other side of the plane, which contains the asymmetric carbon to be examined, the nitrogen and the hydrogen linked at this carbon (Fig. 2). This allowed the assignment of the newly formed asymmetric carbon in $\mathbf{1 0}$ as $R$. This absolute configuration corresponds to the reduction of the imine function of $\mathbf{8 a}$ on the more accessible prochiral face (as opposed to the chiral auxiliary).

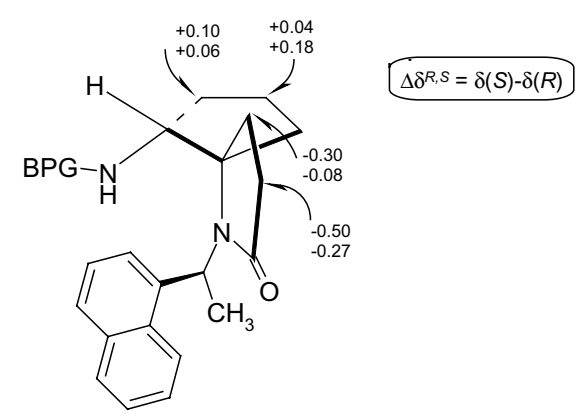

Figure 2.

As a matter of fact, diamine $\mathbf{1 3}$ can be considered as a rigid equivalent of diamine $\mathbf{1}$, described as a good enantioselective catalyst for the reaction of condensation of isovaleraldehyde 17 with $\beta$-nitrostyrene $18 .^{2}$ In order to test the efficiency of chiral diamine $\mathbf{1 3}$ as an 


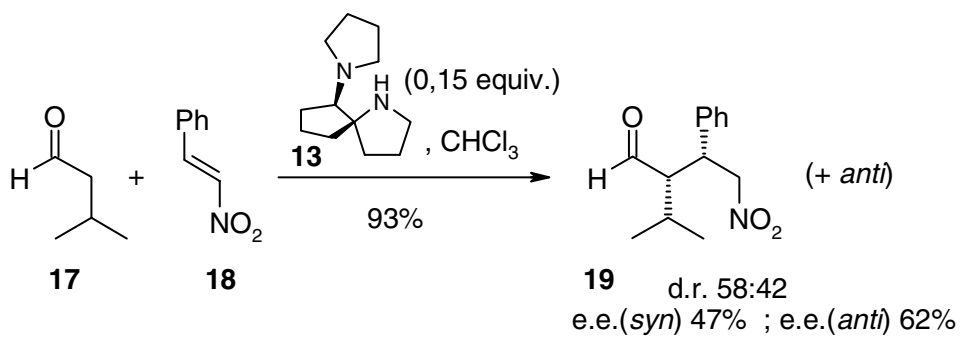

Scheme 4.

enantioselective organic catalyst, we just verified its use in one type of reaction (Scheme 4) under only one set of reaction conditions. Thus, using 0.15 equiv of $\mathbf{1 3}$, following an easy-to-use protocol described by Alexakis and co-workers for diamine $2,{ }^{9}$ the reaction proceeded smoothly to give 19 in $93 \%$ yield. The diastereoselectivity of the condensation was poor (syn/anti 58:42) when compared to pyrrolidine, which gave an 80:20 ratio of synlanti diastereoisomers. On the other hand, the enantioselectivity was encouraging [ee $=47 \%(\mathrm{syn})$ and $62 \%($ anti) ], which can be compared with that obtained with diamine 2 (ee $=61 \%$ for the $s y n$ diastereoisomer). ${ }^{9}$

\section{Conclusion}

The first of a new series of chiral rigid diamines, 6-pyrrolidine-1-yl-1-aza-spiro[4,4]nonane $\mathbf{1 3}$ was easily prepared from 1-azaspiro[4.4]nonane-2,6-dione 4. This compound proved to be an interesting catalyst in an enantioselective reaction. Further studies are currently in progress in order to extend the scope of utilization of this amine. Moreover, starting from the primary amine 10, a large number of new rigid diamines can be synthesized and tested in organic catalyzed reaction.

\section{Experimental section}

\subsection{General}

Moisture sensitive reactions were carried out under an argon atmosphere, using solvents distilled and dried before use by standard methods. Flash chromatographies were carried out on silica gel SDS 60 A C.C 35$70 \mu \mathrm{m} .{ }^{1} \mathrm{H}$ and ${ }^{13} \mathrm{C}$ NMR spectra were performed either with a Bruker-AC300 or a Bruker Avance 400 apparatus. Chemical shifts $(\delta)$ are given in ppm and coupling constant in Hz. IR spectra were performed with a Perkin-Elmer FTIR 1600 apparatus and data are reported in $\mathrm{cm}^{-1}$.

\subsection{Preparation of the chiral diamine 13}

4.2.1. $\left(5 R, 6 R, 1^{\prime} S\right)-6$-Benzylamino-1-(1-naphthalen-1-ylethyl)-1-aza- spiro[4.4]nonan-2-one, 9. To a solution of $4(1.36 \mathrm{~g}, 4.42 \mathrm{mmol})$ in toluene $(22 \mathrm{~mL}, 0.2 \mathrm{M})$ were added benzylamine $(4.8 \mathrm{~mL}, 44.2 \mathrm{mmol})$, and PTSA $(0.21 \mathrm{~g}, 1.1 \mathrm{mmol})$. The reaction mixture was refluxed in a Dean-Stark apparatus for $16 \mathrm{~h}$, cooled to rt, poured into a saturated aqueous solution of $\mathrm{NaHCO}_{3}$, and extracted with $\mathrm{CH}_{2} \mathrm{Cl}_{2}$. The organic phase was dried over $\mathrm{Na}_{2} \mathrm{SO}_{4}$ and concentrated under vacuum, providing a mixture of $\mathbf{8 a}$ and $\mathbf{8 b}(2.5 \mathrm{~g}$, quant.). A solution of crude product in $\mathrm{MeOH}(22 \mathrm{~mL}, 0.2 \mathrm{M})$ was cooled to $0{ }^{\circ} \mathrm{C}$ and $\mathrm{NaBH}_{4}(334 \mathrm{mg}, 8.83 \mathrm{mmol})$ was added portionwise. The solution was stirred for $4 \mathrm{~h}$ reaching $\mathrm{rt}$. Additional $\mathrm{NaBH}_{4}(165 \mathrm{mg}, 4.4 \mathrm{mmol})$ was added and the solution stirred overnight. The mixture was concentrated under vacuum, retaken with water, and extracted with $\mathrm{CH}_{2} \mathrm{Cl}_{2}$. The organic phase was dried over $\mathrm{Na}_{2} \mathrm{SO}_{4}$ and concentrated under vacuum, providing 9 ( $2.45 \mathrm{~g}$, quant.) as a slightly colored amorphous solid. An analytical sample was obtained by flash chromatography $\left(\mathrm{CH}_{2} \mathrm{Cl}_{2}-\mathrm{MeOH} 95: 5\right) . \mathrm{Mp} 150^{\circ} \mathrm{C}$; IR (KBr): $v$ 2973, 2952, 2864, 1680, 1459, 1358, $1304 \mathrm{~cm}^{-1}$; $\mathrm{MS}\left(\mathrm{CI}, \mathrm{NH}_{3}\right): m / z=399\left(\mathrm{MH}^{+}\right) ;{ }^{1} \mathrm{H}$ NMR $(300 \mathrm{MHz}$, $\left.\mathrm{CDCl}_{3}\right): \delta 0.95(\mathrm{~m}, 2 \mathrm{H}), 1.60(\mathrm{~m}, 1 \mathrm{H}), 1.78(\mathrm{~m}, 1 \mathrm{H})$, $1.87-1.96(\mathrm{~m}, 2 \mathrm{H}), 1.98(\mathrm{~d}, J=7.2 \mathrm{~Hz}, 3 \mathrm{H}), 2.10(\mathrm{~m}$, $2 \mathrm{H}), 2.30(\mathrm{~m}, 1 \mathrm{H}), 2.45$ (ddd, $J=6.3,8.3,16.2 \mathrm{~Hz}, 1 \mathrm{H}$ ), $2.73(\mathrm{dd}, J=7.0,10.1 \mathrm{~Hz}, 1 \mathrm{H}), 2.85(\mathrm{~m}, 1 \mathrm{H}), 3.38(\mathrm{AB}$, $J=13.4 \mathrm{~Hz}, \Delta \delta=0.12 \mathrm{ppm}, 2 \mathrm{H}), 5.29(\mathrm{q}, J=7.2 \mathrm{~Hz}$, $1 \mathrm{H}), \quad 6.78(\mathrm{~m}, 2 \mathrm{H}), 7.05-7.15(\mathrm{~m}, 3 \mathrm{H}), 7.36(\mathrm{t}$, $J=7.7 \mathrm{~Hz}, 1 \mathrm{H}), 7.51(\mathrm{t}, J=7.3 \mathrm{~Hz}, 1 \mathrm{H}), 7.57(\mathrm{dt}$, $J=1.2,8.0 \mathrm{~Hz}, 1 \mathrm{H}), 7.73(\mathrm{~d}, J=7.8 \mathrm{~Hz}, 1 \mathrm{H}), 7.92(\mathrm{~m}$, $2 \mathrm{H}), 8.03(\mathrm{~d}, J=7.2 \mathrm{~Hz}, 1 \mathrm{H}) ;{ }^{13} \mathrm{C}$ NMR $(75 \mathrm{MHz}$, $\left.\mathrm{CDCl}_{3}\right): \delta 20.8,21.0,31.0,32.0,34.8,35.7,52.0,52.6$, $68.2,75.1,122.4,125.6,126.2,126.6,126.8,127.8,127.9$, 128.3, 129.6, 130.4, 134.2, 139.4, 140.7, 178.5; HRMS $\left(\mathrm{ESI}^{+},[\mathrm{MH}]^{+}\right): m / z$ calcd 399.2436 , found 399.2433.

4.2.2. (5R,6R,1'S)-6-Amino-1-(1-naphthalen-2-yl-ethyl)1-aza-spiro[4.4|nonan-2-one, 10. To a solution of crude $9(2.45 \mathrm{~g}, \sim 4.42 \mathrm{mmol})$ in $\mathrm{MeOH}(44 \mathrm{~mL}, 0.1 \mathrm{M})$ was added $\mathrm{Pd} / \mathrm{C} 10 \%(850 \mathrm{mg}, \sim 50 \% \mathrm{w} / \mathrm{w})$ under an $\mathrm{N}_{2}$ atmosphere. $\mathrm{N}_{2}$ was replaced by $\mathrm{H}_{2}(1 \mathrm{~atm})$ and the mixture stirred for three days, filtered through a Celite pad, concentrated under vacuum, retaken with a saturated aqueous solution of $\mathrm{NaHCO}_{3}$, and extracted with $\mathrm{CH}_{2} \mathrm{Cl}_{2}$. The organic phase was dried over $\mathrm{Na}_{2} \mathrm{SO}_{4}$ and concentrated under vacuum. This crude product was recrystallized $\left(i-\mathrm{Pr}_{2} \mathrm{O} / \mathrm{EtOH} 95^{\circ} 60: 40\right)$, providing 10 $(250 \mathrm{mg})$ as a white solid. Mother liquor was purified by flash chromatography $\left(\mathrm{CH}_{2} \mathrm{Cl}_{2}-\mathrm{MeOH}-\mathrm{NH}_{4} \mathrm{OH}\right.$ 95:5:0.5) providing additional $\mathbf{1 0}(840 \mathrm{mg}, 81 \%$, three steps). $\mathrm{Mp} 173{ }^{\circ} \mathrm{C} ;[\alpha]_{\mathrm{D}}^{25}=+282$ (c $\left.1.00, \mathrm{CHCl}_{3}\right) ; \mathrm{IR}$ (KBr): $v$ 3381, 2966, 1664, $1361 \mathrm{~cm}^{-1}$; $\mathrm{MS}\left(\mathrm{CI}, \mathrm{NH}_{3}\right)$ : $m / z=309\left(\mathrm{MH}^{+}\right) ;{ }^{1} \mathrm{H}$ NMR $\left(400 \mathrm{MHz}, \mathrm{CDCl}_{3}\right): \delta 0.67$ 
(br s, 2H), $1.02(\mathrm{~m}, 1 \mathrm{H}), 1.55-1.75(\mathrm{~m}, 2 \mathrm{H}), 1.80-1.95$ $(\mathrm{m}, 2 \mathrm{H}), 1.96(\mathrm{~d}, J=7.2 \mathrm{~Hz}, 3 \mathrm{H}), 1.95-2.10(\mathrm{~m}, 2 \mathrm{H})$, $2.33(\mathrm{~m}, 1 \mathrm{H}), 2.47$ (ddd, $J=6.2,9.2,14.8 \mathrm{~Hz}, 1 \mathrm{H}), 2.66$ $(\mathrm{m}, 1 \mathrm{H}), 2.91(\mathrm{t}, J=7.3 \mathrm{~Hz}, 1 \mathrm{H}), 5.39(\mathrm{q}, J=7.1 \mathrm{~Hz}$, $1 \mathrm{H}), 7.48(\mathrm{t}, J=7.6 \mathrm{~Hz}, 2 \mathrm{H}), 7.54(\mathrm{t}, J=7.0 \mathrm{~Hz}, 1 \mathrm{H})$, $7.75(\mathrm{~d}, J=8.1 \mathrm{~Hz}, 1 \mathrm{H}), 7.87(\mathrm{~d}, J=8.0 \mathrm{~Hz}, 1 \mathrm{H}), 7.91$ $(\mathrm{d}, J=8.4 \mathrm{~Hz}, 1 \mathrm{H}), 7.99(\mathrm{~d}, J=7.2 \mathrm{~Hz}, 1 \mathrm{H}) ;{ }^{13} \mathrm{C} \mathrm{NMR}$ $\left(75 \mathrm{MHz}, \mathrm{CDCl}_{3}\right): \delta 20.1,20.8,31.6,33.8,33.9,34.4$, $51.9,62.4,75.1,122.4,125.5,125.6,126.2,126.7,128.0$, 129.6, 130.2, 134.2, 139.2, 178.2. Anal. Calcd for $\mathrm{C}_{20} \mathrm{H}_{24} \mathrm{~N}_{2} \mathrm{O}$ : C, 77.89; H, 7.84; N, 9.08. Found: C, 77.81; $\mathrm{H}, 7.81 ; \mathrm{N}, 9.28$.

4.2.3. $\quad\left(5^{\prime} R, 6^{\prime} R, 1^{\prime \prime} S\right)-1-[1-(1-N a p h t h a l e n-1-y l-e t h y l)-2-$ oxo-1-aza-spiro[4.4]non-6-yl]-pyrrolidine-2,5-dione, 11. To a solution of $10(840 \mathrm{mg}, 2.75 \mathrm{mmol})$ in toluene $(14 \mathrm{~mL}, 0.2 \mathrm{M})$ was added succinic anhydride $(289 \mathrm{mg}$, $2.89 \mathrm{mmol}$ ). The reaction mixture was refluxed for $1.5 \mathrm{~h}$, cooled to rt, concentrated under vacuum, and dissolved in acetyl chloride $(14 \mathrm{~mL}, 0.2 \mathrm{M})$. The reaction mixture was refluxed for $2 \mathrm{~h}$ and concentrated again. Water was added and the mixture extracted with $\mathrm{CH}_{2} \mathrm{Cl}_{2}$. The organic phase was washed with a saturated aqueous solution of $\mathrm{NaHCO}_{3}$, dried over $\mathrm{Na}_{2} \mathrm{SO}_{4}$, and concentrated under vacuum. The crude product was purified by flash chromatography $\left(\mathrm{CH}_{2} \mathrm{Cl}_{2}-\mathrm{MeOH}\right.$ 95:5) providing $11(867 \mathrm{mg}, 81 \%)$ as a white solid. $\mathrm{Mp} 219^{\circ} \mathrm{C}\left(i-\mathrm{Pr}_{2} \mathrm{O}\right)$; $[\alpha]_{\mathrm{D}}^{25}=+71\left(c 1.00, \mathrm{CHCl}_{3}\right)$; IR (KBr): v 2966, 1699, $1686, \quad 1363, \quad 1169 \mathrm{~cm}^{-1} ; \quad \mathrm{MS}\left(\mathrm{CI}, \quad \mathrm{NH}_{3}\right): m / z=391$ $\left(\mathrm{MH}^{+}\right) ;{ }^{1} \mathrm{H}$ NMR $\left(400 \mathrm{MHz}, \mathrm{CDCl}_{3}\right): \delta 1.10(\mathrm{~m}, 4 \mathrm{H})$, $1.65-1.80(\mathrm{~m}, 2 \mathrm{H}), 1.82(\mathrm{~d}, J=7.2 \mathrm{~Hz}, 3 \mathrm{H}), 1.96(\mathrm{~m}$, $1 \mathrm{H}), 2.00-2.20(\mathrm{~m}, 3 \mathrm{H}), 2.22(\mathrm{~m}, 1 \mathrm{H}), 2.41$ (ddd, $J=1.8,9.0,16.3 \mathrm{~Hz}, 1 \mathrm{H}), 2.82(\mathrm{ddd}, J=8.9,11.5$, $16.3 \mathrm{~Hz}, 1 \mathrm{H}), 3.01(\mathrm{~m}, 1 \mathrm{H}), 4.40(\mathrm{~m}, 1 \mathrm{H}), 5.29(\mathrm{q}$, $J=7.1 \mathrm{~Hz}, 1 \mathrm{H}), 7.47(\mathrm{~m}, 2 \mathrm{H}), 7.57(\mathrm{td}, J=7.0,1.1 \mathrm{~Hz}$, $1 \mathrm{H}), 7.62(\mathrm{~d}, J=8.2 \mathrm{~Hz}, 1 \mathrm{H}), 7.73(\mathrm{~d}, J=8.1 \mathrm{~Hz}, 1 \mathrm{H})$, 7.85 (m, 2H); ${ }^{13} \mathrm{C}$ NMR (75 MHz, $\left.\mathrm{CDCl}_{3}\right): \delta 20.5,21.1$, 26.0, 27.4, 30.3, 36.9, 37.1, 50.8, 56.0, 76.0, 123.5, 125.8, $126.3,126.6,127.7,129.2,130.1,133.7,138.1,178.3$, 178.3; HRMS $\left(\mathrm{ESI}^{+},[\mathrm{MH}]^{+}\right): m / z$ calcd 391.2022, found 391.2019 .

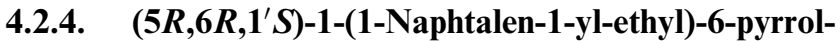
idin-1-yl-1-aza-spiro[4.4]nonan-2-one, 12 . To a $1 \mathrm{M}$ solution of $\mathrm{BH}_{3} \cdot \mathrm{THF}$ in THF was added $11(220 \mathrm{mg}$, $0.56 \mathrm{mmol}$ ). The reaction mixture was refluxed for $16 \mathrm{~h}$ and cooled to $\mathrm{rt}$ after which $\mathrm{MeOH}(1 \mathrm{~mL})$ was slowly added. The reaction mixture was stirred for $15 \mathrm{~min}$, poured into a large quantity of brine, and extracted with $\mathrm{CH}_{2} \mathrm{Cl}_{2}$. Organic phase was dried over $\mathrm{Na}_{2} \mathrm{SO}_{4}$ and concentrated under vacuum providing $\mathbf{1 2}(867 \mathrm{mg}$, quant.) as a white amorphous solid. $[\alpha]_{\mathrm{D}}^{25}=+107$ (c 1.1, $\mathrm{CHCl}_{3}$ ); IR (KBr): $v$ 3048, 2968, 2885, 2442, 2391, 2121, $1458 \mathrm{~cm}^{-1}$; MS(CI, $\left.\mathrm{NH}_{3}\right): \mathrm{m} / z=349\left(\mathrm{MH}^{+}\right) ;{ }^{1} \mathrm{H}$ NMR $\left(400 \mathrm{MHz}, \mathrm{CDCl}_{3}\right): \delta 1.45-1.80(\mathrm{~m}, 3 \mathrm{H}), 1.75(\mathrm{~d}$, $J=7.2 \mathrm{~Hz}, 3 \mathrm{H}), 1.80-2.00(\mathrm{~m}, 2 \mathrm{H}), 2.00-2.15(\mathrm{~m}, 2 \mathrm{H})$, 2.15-2.50 (m, 6H), $2.62(\mathrm{~m}, 1 \mathrm{H}), 2.96(\mathrm{~m}, 1 \mathrm{H}), 3.05-3.25$ (m, 3H), $3.59(\mathrm{~m}, 1 \mathrm{H}), 3.78(\mathrm{~m}, 1 \mathrm{H}), 4.45(\mathrm{~m}, 1 \mathrm{H}), 5.52$ $(\mathrm{q}, J=7.2 \mathrm{~Hz}, 1 \mathrm{H}), 7.35-7.55(\mathrm{~m}, 2 \mathrm{H}), 7.63 \quad(\mathrm{t}$, $J=8.2 \mathrm{~Hz}, 1 \mathrm{H}), 7.76(\mathrm{~d}, J=7.3 \mathrm{~Hz}, 1 \mathrm{H}), 7.80-7.90(\mathrm{~m}$, $2 \mathrm{H}), 8.10(\mathrm{~d}, J=8.6 \mathrm{~Hz}, 1 \mathrm{H}) ;{ }^{13} \mathrm{C}$ NMR $(75 \mathrm{MHz}$,
$\left.\mathrm{CDCl}_{3}\right): \delta 20.6,21.7,22.2,22.3,23.8,25.8,34.4,38.0$, $57.9,58.7,64.4,60.7,77.7,89.4,122.7,125.5,125.9$, $126.2,127.5,129.7,130.0,131.5,134.2,135.3$; HRMS $\left(\mathrm{ESI}^{+},[\mathrm{MH}]^{+}\right): m / z$ calcd 349.2644 , found 349.2648 .

4.2.5. (5R,6R)-6-Pyrrolidin-1-yl-1-aza-spiro[4.4]nonane, 13. To a solution of crude $12(102 \mathrm{mg}, \sim 0.26 \mathrm{mmol})$ in $\mathrm{MeOH}(3 \mathrm{~mL}, 0.1 \mathrm{M})$ was added $\mathrm{Pd} / \mathrm{C} 10 \%(50 \mathrm{mg}$, $\sim 50 \%$ w/w) under an $\mathrm{N}_{2}$ atmosphere. $\mathrm{N}_{2}$ was replaced by $\mathrm{H}_{2}(1 \mathrm{~atm})$ and the mixture stirred for $16 \mathrm{~h}$, filtered through a Celite pad, concentrated under vacuum, and retaken with a $1 \mathrm{M}$ aqueous solution of $\mathrm{HCl}$. This aqueous phase was washed with $\mathrm{CH}_{2} \mathrm{Cl}_{2}$, basified by $15 \%$ aqueous solution of $\mathrm{NaOH}$, and extracted with $\mathrm{Et}_{2} \mathrm{O}$. Organic phase was dried over $\mathrm{Na}_{2} \mathrm{SO}_{4}$ and concentrated under vacuum, providing $13(38 \mathrm{mg}, 76 \%$, two steps) as a colorless oil. ${ }^{1} \mathrm{H}$ NMR $\left(400 \mathrm{MHz}, \mathrm{CDCl}_{3}\right): \delta$ 1.45-1.63 (m, 4H), 1.65-1.95 (m, 10H), 2.45-2.60 (m, $6 \mathrm{H}), 2.71(\mathrm{~m}, 1 \mathrm{H}), 3.05(\mathrm{~m}, 1 \mathrm{H}) ;{ }^{13} \mathrm{C}$ NMR $(75 \mathrm{MHz}$, $\left.\mathrm{CDCl}_{3}\right): \delta 20.7,23.7,27.5,30.8,36.6,41.1,46.8,53.4$, 71.5, 77.4. IR (KBr): $v$ 3278, 2956, 2870, 2785, 1458, $1415,1343 \mathrm{~cm}^{-1}$; HRMS $\left(\mathrm{ESI}^{+},[\mathrm{MH}]^{+}\right): \mathrm{m} / z$ calcd 195.1861, found 195.1867 .

13-Picric acid (3/2): $\mathrm{mp} 185^{\circ} \mathrm{C}(\mathrm{EtOH}) ;[\alpha]_{\mathrm{D}}^{25}=-48(c$ $\left.0.4, \mathrm{CHCl}_{3}\right)$.

4.2.6. $\left(1 R, 6^{\prime} R, 1^{\prime \prime} R\right)-\{[1-(1-N a p h t h a l e n-2-y l-e t h y l)-2-o x 0-$ 1-aza-spiro[4.4]non-6-ylcarbamoyl]-phenyl-methyl\}-carbamamic acid tert-butyl ester, 14a. Spirodiamine 13 $(50 \mathrm{mg}, \quad 0.16 \mathrm{mmol})$ and $(R)-N$-Boc-phenylglycine $(40.7 \mathrm{mg}, 0.16 \mathrm{mmol})$ were dissolved in dry $\mathrm{CH}_{2} \mathrm{Cl}_{2}$ $(1 \mathrm{~mL}, c=0.16 \mathrm{M})$ under an argon atmosphere. The solution was cooled to $0^{\circ} \mathrm{C}$ and $N, N^{\prime}$-dicyclohexylcarbodiimide $(37 \mathrm{mg}, 0.18 \mathrm{mmol})$ added in one drop. The mixture was allowed to warm to $\mathrm{rt}$ and stirred at this temperature for $90 \mathrm{~min}$. The white precipitate, which formed, was filtered and washed with $\mathrm{CH}_{2} \mathrm{Cl}_{2}$. The solvent was evaporated to dryness and the crude reaction mixture purified by flash chromatography on silica gel (AcOEt-cyclohexane 60:40) to provide 14a $(80 \mathrm{mg}$, $91 \%$ as a white solid. $\mathrm{Mp} 153-155^{\circ} \mathrm{C}$ (EtOH); $[\alpha]_{\mathrm{D}}^{20}=+203\left(c\right.$ 1.03, $\mathrm{CHCl}_{3}$ ); IR (film): v 3387, 3058, 2973, 2934, 2884, 1714, 1682, 1510, 1367; ${ }^{1} \mathrm{H}$ NMR $\left(400 \mathrm{MHz}, \mathrm{CDCl}_{3}\right): \delta 0.96(\mathrm{~m}, 1 \mathrm{H}), 1.46(\mathrm{~s}, 9 \mathrm{H}), 1.73(\mathrm{~m}$, $2 \mathrm{H}), 1.93(\mathrm{~m}$ with d, $J=7.1 \mathrm{~Hz}, 2 \mathrm{H}), 1.95(\mathrm{~m}, 1 \mathrm{H}), 2.00$ $(\mathrm{m}, 1 \mathrm{H}), 2.04(\mathrm{~m}, 1 \mathrm{H}), 2.16(\mathrm{~m}, 1 \mathrm{H}), 2.28(\mathrm{~m}, 1 \mathrm{H}), 4.06$ $(\mathrm{m}, 1 \mathrm{H}), 4.68($ br s, $1 \mathrm{H}), 5.14(\mathrm{q}, J=7.1 \mathrm{~Hz}, 1 \mathrm{H}), 5.29$ $(\mathrm{d}, J=5.4 \mathrm{~Hz}, 1 \mathrm{H}), 5.66(\mathrm{~d}, J=8.0 \mathrm{~Hz}, 1 \mathrm{H}), 6.97(\mathrm{~m}$, $2 \mathrm{H}), \quad 7.25(\mathrm{~m}, 3 \mathrm{H}), 7.45-7.65(\mathrm{~m}, 3 \mathrm{H}), 7.81(\mathrm{t}$, $J=7.2 \mathrm{~Hz}, 2 \mathrm{H}), 7.89(\mathrm{dd}, J=1.5,7.0 \mathrm{~Hz}, 1 \mathrm{H}), 7.98(\mathrm{~d}$, $J=6.7 \mathrm{~Hz}, 1 \mathrm{H}) ;{ }^{13} \mathrm{C}$ NMR $\left(75 \mathrm{MHz}, \mathrm{CDCl}_{3}\right): \delta 20.7$, 21.1, 28.6, 31.0, 33.1, 36.4, 51.7, 58.8, 59.7, 74.2, 80.7, $121.7,124.9,126.3,127.2,127.4,128.9,129.4,129.9$, $130.2,134.3,138.0,140.2,154.9,170.6,178.0$; HRMS $\left(\mathrm{ESI}^{+},[\mathrm{MH}]^{+}\right): m / z$ calcd 564.2838, found 564.2818.

4.2.7. $\left(1 S, 6^{\prime} R, 1^{\prime \prime} R\right)-\{[1-(1-N a p h t h a l e n-2-y l-e t h y l)-2-0 x 0-$ 1-aza-spiro[4.4]non-6-ylcarbamoyl]-phenyl-methyl\}-carbamamic acid tert-butyl ester, $14 \mathrm{~b}$. The same procedure 
as above furnished $\mathbf{1 4 b}$ in a quantitative yield $(75 \mathrm{mg})$ starting from $13(42 \mathrm{mg}, 0.14 \mathrm{mmol})$ and $(S)-N$-Bocphenylglycine $(35.5 \mathrm{mg}, 0.14 \mathrm{mmol}) . \mathrm{Mp} \quad 105-108^{\circ} \mathrm{C}$ $\left(\right.$ EtOH); $[\alpha]_{\mathrm{D}}^{20}=+279\left(c 1.00, \mathrm{CHCl}_{3}\right)$; IR (film): $v 3386$, $3058,2974,2936,2885,1714,1683,1510,1369 ;{ }^{1} \mathrm{H}$ NMR $\left(400 \mathrm{MHz}, \mathrm{CDCl}_{3}\right): \delta 0.92(\mathrm{~m}, 1 \mathrm{H}), 1.39(\mathrm{~s}, 9 \mathrm{H})$, $1.55(\mathrm{~m}, 1 \mathrm{H}), 1.63(\mathrm{~m}, 1 \mathrm{H}), 1.89(\mathrm{~m}, 1 \mathrm{H}), 1.96(\mathrm{~d}$, $J=7.1 \mathrm{~Hz}, 1 \mathrm{H}), 2.04(\mathrm{~m}, 1 \mathrm{H}), 2.08(\mathrm{~m}, 1 \mathrm{H}), 2.24(\mathrm{~m}$, $1 \mathrm{H}), 2.35(\mathrm{~m}, 1 \mathrm{H}), 2.43(\mathrm{~m}, 2 \mathrm{H}), 4.14(\mathrm{~m}, 1 \mathrm{H}), 4.33(\mathrm{br} \mathrm{s}$, $1 \mathrm{H}), 5.08(\mathrm{br} \mathrm{s}, 1 \mathrm{H}), 5.12(\mathrm{q}, J=7.1 \mathrm{~Hz}, 1 \mathrm{H}), 5.56(\mathrm{br} \mathrm{s}$, $1 \mathrm{H}), 6.76$ (br s, 2H), 7.00-7.10 (m, 3H), $7.55(\mathrm{~m}, 2 \mathrm{H})$, $7.63(\mathrm{t}, J=7.8 \mathrm{~Hz}, 1 \mathrm{H}), 7.85(\mathrm{~m}, 2 \mathrm{H}), 7.94(\mathrm{~m}, 1 \mathrm{H}), 8.14$ $(\mathrm{dd}, J=0.8,7.2 \mathrm{~Hz}, 1 \mathrm{H}) ;{ }^{13} \mathrm{C}$ NMR $\left(75 \mathrm{MHz}, \mathrm{CDCl}_{3}\right): \delta$ $20.8,21.0,28.6,31.6,31.8,33.9,36.5,51.9,58.9$ (2C), $74.4,80.2,121.7,125.7,126.1,126.7,127.3,128.3,128.9$, $130.1,130.1,134.3,138.2,139.2,154.8,170.5,178.2$; HRMS $\left(\mathrm{ESI}^{+},[\mathrm{MH}]^{+}\right): \mathrm{m} / z$ calcd 564.2838, found 564.2838 .

\subsection{Michael addition reaction}

To a mixture of propionaldehyde (10 equiv) and either pyrrolidine or diamine 13 ( 0.15 equiv) in chloroform $(0.1 \mathrm{M})$ was added nitrostyrene (1 equiv). The mixture was stirred at $\mathrm{rt}$ for three days. The reaction was quenched at $0{ }^{\circ} \mathrm{C}$ with a $1 \mathrm{M}$ aqueous solution of $\mathrm{HCl}$ and extracted with $\mathrm{CH}_{2} \mathrm{Cl}_{2}$. The organic phase was dried over $\mathrm{Na}_{2} \mathrm{SO}_{4}$, concentrated under vacuum, and purified by flash chromatography, providing $19(93-95 \%)$ as a colorless oil. A sample of each pure diastereoisomer was then obtained. Analytical data are identical as those described. ${ }^{9 a}$ Enantiomeric excesses have been determined by chiral HPLC (Chiralpak AD, hexane- $i-\mathrm{PrOH}$ 90:10).

\section{Acknowledgements}

This research was supported by the C.N.R.S. and the M.E.N.R.T.

\section{References and notes}

1. For a review, see: Lucet, D.; LeGall, T.; Mioskowski, C. Angew. Chem., Int. Ed. Engl. 1998, 37, 2581-2627.

2. For a review, see: Magnus, A.; Bertilsson, S. K.; Andersson, P. G. Chem. Soc. Rev. 2002, 31, 223-229.

3. For recent examples, see: (a) Li, X.; Chen, W.; Hems, W.; King, F.; Xiao, J. Org. Lett. 2003, 5, 4559-4561; (b) Li, X.; Chen, W.; Hems, W.; King, F.; Xiao, J. Tetrahedron Lett. 2004, 45, 951-953; (c) Hamada, T.; Manabe, K.; Kobayashi, S. Angew. Chem., Int. Ed. Engl. 2003, 42, 3927-3930; (d) Belokon, Y. N.; Fuentes, J.; North, M.; Steed, J. W. Tetrahedron 2004, 60, 3191-3204; (e) Kobayashi, S.; Matsubara, R.; Nakamura, Y.; Kitagawa, H.; Sugiura, M. J. Am. Chem. Soc. 2003, 125, 2507-2515; (f) Bette, V.; Mortreux, A.; Savoia, D.; Carpentier, J.-F. Tetrahedron 2004, 60, 2837-2842.

4. For recent example, see: Shiina, I.; Oshiumu, H.; Hashizume, M.; Yamai, Y.-s.; Ibuka, R. Tetrahedron Lett. 2004, 45, 543-547.

5. (a) Dalko, P. I.; Moisan, L. Angew. Chem., Int. Ed. Engl. 2001, 40, 3726-3748; (b) France, S.; Guerin, D. J.; Miller, S. J.; Lectka, T. Chem. Rev. 2003, 103, 2985-3012.

6. (a) Beak, P.; Basu, A.; Gallagher, D. J.; Park, Y. S.; Thayumanavan, S. Acc. Chem. Res. 1996, 29, 552-560; (b) Hoppe, D.; Hense, T. Angew. Chem., Int. Ed. Engl. 1997, 36, 2282-2316.

7. With the exception of cinchona alkaloids quinine and quinidine.

8. Betancort, J. M.; Barbas, C. F., III. Org. Lett. 2001, 3, 3737-3740.

9. (a) Alexakis, A.; Andrey, O. Org. Lett. 2002, 4, 3611-3614; (b) Andrey, O.; Alexakis, A.; Bernadinelli, G. Org. Lett. 2003, 5, 2959-2961.

10. Planas, L.; Pérard-Viret, J.; Royer, J.; Selkti, M.; Tomas, A. Synlett 2002, 1629-1632.

11. (a) Baussanne, I.; Chiaroni, A.; Husson, H.-P.; Riche, C.; Royer, J. Tetrahedron Lett. 1994, 35, 3931-3934; (b) Baussanne, I.; Travers, C.; Royer, J. Tetrahedron: Asymmetry 1998, 9, 797-804; (c) Dudot, B.; Chiaroni, A.; Royer, J. Tetrahedron Lett. 2000, 41, 6355-6359; (d) Baussanne, I.; Chiaroni, A.; Royer, J. Tetrahedron: Asymmetry 2001, 12, 1219-1224.

12. Seco, J. M.; Quinoa, E.; Riguera, R. J. Org. Chem. 1999, 64, 4669-4675. 